ESAIM: M2AN 48 (2014) 1859-1876

DOI: $10.1051 / \mathrm{m} 2 \mathrm{an} / 2014022$
ESAIM: Mathematical Modelling and Numerical Analysis

www.esaim-m2an.org

\title{
MOVING DIRICHLET BOUNDARY CONDITIONS *
}

\author{
Robert Altmann ${ }^{1}$
}

\begin{abstract}
This paper develops a framework to include Dirichlet boundary conditions on a subset of the boundary which depends on time. In this model, the boundary conditions are weakly enforced with the help of a Lagrange multiplier method. In order to avoid that the ansatz space of the Lagrange multiplier depends on time, a bi-Lipschitz transformation, which maps a fixed interval onto the Dirichlet boundary, is introduced. An inf-sup condition as well as existence results are presented for a class of second order initial-boundary value problems. For the semi-discretization in space, a finite element scheme is presented which satisfies a discrete stability condition. Because of the saddle point structure of the underlying PDE, the resulting system is a DAE of index 3.
\end{abstract}

Mathematics Subject Classification. 65J10, 65M60, 65M20.

Received September 25, 2013. Revised March 16, 2014

Published online October 10, 2014.

\section{INTRODUCTION}

Consider an initial-boundary value problem as it arises in many applications in which a dynamic behavior is modeled. No matter which particular problem is analyzed, initial and boundary values are needed in order to obtain a well-posed problem. Although the boundary conditions may depend on time, the part of the boundary on which they are specified is usually fixed. In this paper, we analyse Dirichlet boundary conditions on a timedependent boundary part $\Gamma_{D}(t)$. A simple example is shown in Figure 1. Therein, an elastic body $\Omega$ is coupled through Dirichlet boundary conditions with a spring damper system, which moves to the right with a given speed $v_{0}$.

In a more general framework, the here presented model can be used to couple problems from different physics or to model flexible multibody systems [18,28]. Consider for example the pantograph and catenary dynamics [27] analyzed in [5]. This one-dimensional benchmark problem contains a coupling of partial differential equations (PDE) and differential-algebraic equations (DAE). The critical part of this model is the contact between pantograph and catenary to achieve the transmission of electrical energy. In the mentioned model $[4,5]$, the contact is modeled by unilateral constraints and thus, actually given by inequalities. This can be treated by slack variables or barrier functions [13]. Then, the contact constraint is given in the form

$$
u\left(x_{p}(t), t\right)=g(t) .
$$

Keywords and phrases. Dirichlet boundary conditions, operator DAE, inf-sup condition, wave equation.

* The author's work was supported by the ERC Advanced Grant "Modeling, Simulation and Control of Multi-Physics Systems" MODSIMCONMP and the Berlin Mathematical School BMS.

1 Institut für Mathematik MA4-5, Technische Universität Berlin, Straße des 17. Juni 136, 10623 Berlin, Germany.

raltmann@math.tu-berlin.de 


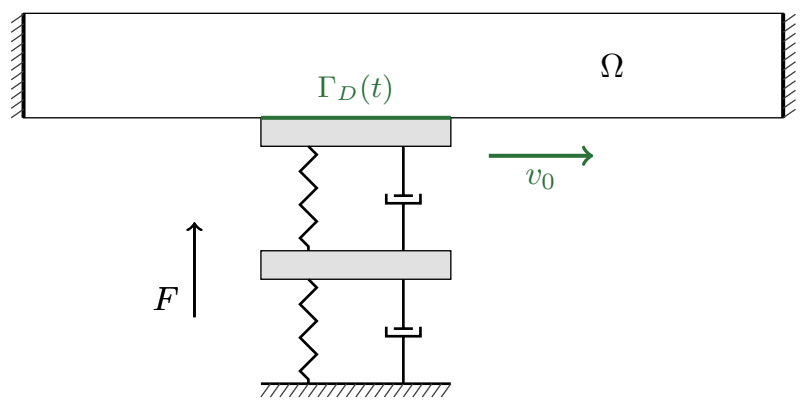

FIGURE 1. Example of a flexible body $\Omega$ coupled with a spring damper system along $\Gamma_{D}(t) \subseteq$ $\partial \Omega$. The spring damper system moves to the right with speed $v_{0}$. In addition, a force $F$ acts upwards on the spring damper.

Therein, $x_{p}(t)$ is the position of pantograph, $g(t)$ its height, and $u$ the deformation of the wire. Because of well-known embedding theorems ([16], Chap. 5), point constraints are only well-defined for one-dimensional problems. But even in this case they lead to numerical instabilities such that the contact constraint is typically modeled via a regularized point constraint including a regularized delta distribution.

Assume that we model the wire of the catenary in a more detailed way, for example by a two-dimensional model. In this case, the pantograph has to be in contact with the boundary of the wire. Since the train is moving with a certain speed, the coupling constraint has the form of a moving Dirichlet boundary condition. Note that the example in Figure 1 is a strongly simplified model of the pantograph and catenary system. The spring damper system represents the pantograph which acts with a force $F$ upwards to stay in contact with the wire.

In the here presented model, the moving Dirichlet boundary conditions are incorporated in form of a weak constraint via the Lagrange multiplier method $[6,7,10]$. Since the boundary conditions are intended to model coupling constraints, they should not be included in the ansatz space of the deformation, as suggested in many PDE text books (e.g. [11], Chap. 5.4). This already accounts for the coupling of flexible bodies through fixed Dirichlet boundaries since the deformation along the boundary may depend on the motion of adjacent bodies $[29,30]$. This modeling procedure leads to a dynamic, also called transient, saddle point problem. The structure is then similar to the ansatz used for mortar methods [8].

In this paper, we aim to formulate a framework to incorporate Dirichlet conditions on moving boundary parts. Since we enforce the boundary conditions as a weak constraint, we require a suitable ansatz space for the Lagrange multiplier. In order to avoid a time-dependent ansatz space, the model is based on a bi-Lipschitz transformation of the moving Dirichlet boundary. With this transformation, we can introduce a constraint operator which satisfies the usual stability condition. This allows to formulate existence results of solutions for the resulting constrained operator system.

Because of the saddle point structure, capable finite element spaces for the discretization in space lead to DAEs of (differentiation) index 3. For a definition and a review of the various index concepts of DAEs, we refer to Chapter 1.2 from [21].

The paper is organized as follows. In Section 2 a time-dependent bi-Lipschitz transformation is introduced, which maps a fixed interval onto the moving Dirichlet boundary part. With this transformation we can formulate the constrained operator equations of motion. The section ends with a discussion on the existence of solutions for second order initial-boundary value problems and in particular for the linear wave equation.

The spatial discretized equations are subject of Section 3. We apply piecewise linear and globally continuous finite elements combined with edge-bubble functions. Together with a piecewise constant discretization of the Lagrange multiplier, this yields under certain conditions a stable discretization scheme in the sense of a discrete inf-sup condition. In Section 4 we close with some concluding remarks. 
Throughout this paper, we write $\left.v\right|_{\partial \Omega}$ for the image of the trace operator applied to $v \in H^{1}(\Omega)$, which has a well-defined trace ([31], Chap. 2.5). Furthermore, we write $A \lesssim B$ if there exists a generic constant $c>0$ with $A \leq c B$. This constant is independent of the mesh-size and time. Finally, for an edge $E$ we denote its length by $|E|$.

\section{Continuous model}

Thinking primarily about problems from elastodynamics, we discuss second order initial-boundary value problems. Nevertheless, the presented method can be applied to first order systems as well. Consider the second order initial-boundary value problem in operator form

$$
\mathcal{M} \ddot{u}(t)+\mathcal{D} \dot{u}(t)+\mathcal{K} u(t)=\mathcal{F}(t)
$$

for $t \in\left(t_{0}, T\right]$ with initial conditions for $u\left(t_{0}\right), \dot{u}\left(t_{0}\right)$ and Dirichlet boundary conditions of the form

$$
u(t)=u_{D}(t) \quad \text { on } \Gamma_{D}(t) \subset \partial \Omega .
$$

In the dynamics of elastic media, the operator $\mathcal{M}$ includes the density of the investigated material. The operator $\mathcal{K}$ incorporates the stiffness, i.e., a possibly nonlinear material law, and $\mathcal{D}$ a viscous damping term. This framework includes the wave equation, vibrating membranes [23] as well as examples from nonconvex elastodynamics modeling shape memory alloys [15].

As mentioned in the introduction, we include the Dirichlet boundary condition (2.1) on the time-dependent boundary part $\Gamma_{D}(t)$ in form of a constraint since $u_{D}$ might be unknown a priori [29]. In operator form, the Dirichlet boundary condition reads $\mathcal{B}(t) u(t)=\mathcal{G}(t)$ with the linear operator $\mathcal{B}(t)$ defined in Section 2.4 below. Adding this constraint to the system, we have to introduce a Lagrange multiplier [6]. The derivation of a suitable ansatz space is subject of the following subsection.

In the sequel, we denote the Sobolev space on a domain $D$ of order $\alpha$ by $H^{\alpha}(D)$, see [1] for an introduction. The corresponding norm is denoted by $\|\cdot\|_{\alpha, D}$. This includes the $L^{2}$-norm (which equals $H^{0}$ ) as well as negative norms,

$$
\|\cdot\|_{\alpha, \Omega}:=\|\cdot\|_{H^{\alpha}(\Omega)}, \quad\|\cdot\|_{0, \Omega}:=\|\cdot\|_{L^{2}(\Omega)}, \quad\|\cdot\|_{-1 / 2, \Gamma}:=\|\cdot\|_{\left[H^{1 / 2}(\Gamma)\right]^{*}} \cdot
$$

\subsection{Preliminaries}

Let $\Omega \subset \mathbb{R}^{n}$ denote an open, bounded, and connected domain with Lipschitz boundary $\partial \Omega$ [9], Chapter I. We assume that the time-dependent part of the boundary on which we have Dirichlet boundary conditions has positive measure and is denoted by $\Gamma_{D}(t) \subset \partial \Omega$. Furthermore, we assume that $\Gamma_{D}(t)$ changes continuously in time which may include a change of length.

Depending on the underlying initial-boundary value problem, the solution is a time-dependent mapping from $\Omega$ to $\mathbb{R}^{d}$. Considering the wave equation, we seek for the velocity $u(t): \Omega \rightarrow \mathbb{R}$, i.e., $d=1$. In the case of elastodynamics, the unknown is the deformation in every space direction and thus, $d=n$. As search space for the deformation (respectively velocity) we introduce the space of square integrable functions in $d$ components, which also have a square integrable weak derivative,

$$
\mathcal{V}:=\left[H^{1}(\Omega)\right]^{d} .
$$

By $\mathcal{V}^{*}$ we denote its dual space. In order to shorten notation, we introduce the space

$$
\mathcal{H}:=\left[L^{2}(\Omega)\right]^{d}
$$

Note that $\mathcal{V}, \mathcal{H}, \mathcal{V}^{*}$ form a Gelfand triple [34], Chapter 23.4. As a consequence, $v \in \mathcal{V}$ is embedded in $\mathcal{V}^{*}$ such that for all $w \in \mathcal{V}$,

$$
\langle v, w\rangle_{\mathcal{V}^{*}, \mathcal{V}}=(v, w)_{\mathcal{H}}
$$


Therein, $\langle\cdot, \cdot\rangle_{\mathcal{V}^{*}, \mathcal{V}}$ denotes the duality pairing of $\mathcal{V}^{*}, \mathcal{V}$ and $(\cdot, \cdot)_{\mathcal{H}}$ the inner product in $\mathcal{H}$. Furthermore, we have the continuous embedding [34], Chapter 23.6,

$$
\left\{u \in L^{2}\left(t_{0}, T ; \mathcal{V}\right) \mid \dot{u} \in L^{2}\left(t_{0}, T ; \mathcal{V}^{*}\right)\right\} \hookrightarrow C\left(\left[t_{0}, T\right] ; \mathcal{H}\right) .
$$

It remains to find a suitable ansatz space of the Lagrange multiplier. If the Dirichlet boundary is independent of time, the natural ansatz space for the Lagrange multiplier is the dual space of the broken Sobolev space $H^{1 / 2}\left(\Gamma_{D}\right)$, see $[2,29]$. For a definition of broken Sobolev spaces, we refer to [1]. However, the right choice for the dynamic case is not clear since a direct adoption would lead to the dual space of $H^{1 / 2}\left(\Gamma_{D}(t)\right)$ which is time-dependent. Such a space would cause difficulties in the modeling process and also within the discretization procedure.

A solution to this problem is the introduction of a bi-Lipschitz transformation which maps a fixed, i.e., time-independent, $(n-1)$-dimensional domain $I$ with positive Lebesgue measure onto the Dirichlet boundary $\Gamma_{D}(t)$. More details on needed assumptions are given in the following subsection. We then define the Lagrange multiplier on $I$. For this, we define a Hilbert space $\mathcal{Q}$ via its dual space,

$$
\mathcal{Q}^{*}:=\left[H^{1 / 2}(I)\right]^{d} .
$$

Remark 2.1. Since $\mathcal{Q}^{*}$ is densely embedded in $\left[L^{2}(I)\right]^{d}$, the three spaces $\mathcal{Q}^{*},\left[L^{2}(I)\right]^{d}, \mathcal{Q}$ form a Gelfand triple. Thus, the duality pairing of $\mathcal{Q}^{*}, \mathcal{Q}$ is densely defined by the $L^{2}$ inner product on $I$.

Recall that the Hilbert space $H^{1 / 2}(I)$ contains the traces of $H^{1}$-functions. Thus, an inner product can be defined by the inner product in $H^{1}$ of the solutions of corresponding homogeneous Dirichlet problems ([12], Chap. III.1). An alternative approach can be found in Chapter I.7.3 from [22].

\subsection{Bi-Lipschitz transformation}

This section is devoted to the transformation which maps the time-independent $(n-1)$-dimensional domain $I$ onto $\Gamma_{D}(t)$. Clearly, the transformation has to be time-dependent. We introduce

$$
\Phi:\left[t_{0}, T\right] \times \mathbb{R}^{n} \rightarrow \mathbb{R}^{n}
$$

and require the following properties. For every $t \in\left[t_{0}, T\right]$ we assume $\Phi(t): \mathbb{R}^{n} \rightarrow \mathbb{R}^{n}$ to be a bi-Lipschitz transformation, i.e., the function is bijective and $\Phi(t)$ as well as $\Phi^{-1}(t)$ are Lipschitz continuous. Thus, by Rademacher's theorem ([16], Chap. 5.8), $\Phi(t)$ and its inverse are differentiable a.e. in $\mathbb{R}^{n}$. We denote this derivative by $\mathrm{D} \Phi(t)$ and assume that it is (w.r.t. a $n$-dimensional measure) a.e. uniformly bounded in $t$, i.e., there exist constants $0<c_{\Phi}<C_{\Phi}<\infty$ with

$$
c_{\Phi} \leq|\operatorname{det} \mathrm{D} \Phi(t)| \leq C_{\Phi} .
$$

Clearly, also $\left|\operatorname{det} \mathrm{D} \Phi^{-1}(t)\right|$ is a.e. bounded by the constants $C_{\Phi}^{-1}$ and $c_{\Phi}^{-1}$. In particular, we assume (2.3) to hold (w.r.t. a $(n-1)$-dimensional measure) a.e. on $I$.

Remark 2.2. The boundedness of $\mathrm{D} \Phi(t)$ clearly limits the length evolution of the Dirichlet boundary. This restriction is necessary since the Dirichlet boundary has to be of positive measure to ensure the well-posedness of the problem.

Since the Dirichlet boundary $\Gamma_{D}(t)$ moves continuously with respect to time, we assume that $\Phi$ is continuous in $t$ as well. Recall that we introduce a time-dependent transformation in order to map $I$ onto $\Gamma_{D}(t)$. Therefore, we assume that

$$
\left.\Phi(t)\right|_{I}: I \rightarrow \Gamma_{D}(t)
$$


is onto. The last requirement for $\Phi$ concerns the inverse image of $\Omega$. Since $\Phi(t)$ is bijective, we are able to define $\Sigma(t)$ as the domain satisfying

$$
\Phi(t, \Sigma(t))=\Omega
$$

For fixed $t \in\left[t_{0}, T\right]$, we may also define the Sobolev space on $\Sigma(t),\left[H^{1}(\Sigma(t))\right]^{d}$. Since $I$ is mapped onto $\Gamma_{D}(t) \subset \partial \Omega$ and thus $I \subset \partial \Sigma(t)$, the inverse trace theorem ([31], Thm. 2.22) gives a continuous map of the form

$$
\mathcal{Q}^{*} \rightarrow\left[H^{1}(\Sigma(t))\right]^{d}
$$

The involved continuity constant depends on the domain $\Sigma(t)$ and therefore on time.

Assumption 2.3 (Uniform inverse trace constant). Let $C_{\mathrm{inv} \operatorname{Tr}(\Sigma(t))}$ denote the continuity constant given by the inverse trace theorem with respect to $\mathcal{Q}^{*}$ and $\left[H^{1}(\Sigma(t))\right]^{d}$. We assume that these constants are uniformly bounded in $t$ by a constant $C_{\text {invTr }}$, i.e., for all $t \in\left[t_{0}, T\right]$ we have that

$$
C_{\mathrm{inv} \operatorname{Tr}(\Sigma(t))} \leq C_{\mathrm{invTr}} .
$$

Remark 2.4. Assumption 2.3 is certainly fulfilled if $\Sigma(t)$ is independent of $t$, i.e., the inverse image of $\Omega$ under $\Phi(t)$ is a fixed domain. The same is true in the case where $\Sigma(s)$ and $\Sigma(t)$ only differ by a translation for all $s, t \in\left[t_{0}, T\right]$.

Theorem 2.5 (Bi-Lipschitz equivalence). Consider two domains $\Sigma, \Omega$ and a bi-Lipschitz transformation $\Phi$ with $\Phi(\Sigma)=\Omega$ and $\Phi\left(\Gamma_{\Sigma}\right)=\Gamma_{\Omega}$ for boundary parts $\Gamma_{\Sigma} \subseteq \partial \Sigma$ and $\Gamma_{\Omega} \subseteq \partial \Omega$. Then, the operators

$$
\begin{aligned}
A_{1}: & H^{1}(\Omega) \\
u & \rightarrow H^{1}(\Sigma) \\
u & \mapsto u \circ \Phi, \\
A_{1 / 2}: & H^{1 / 2}\left(\Gamma_{\Omega}\right) \rightarrow H^{1 / 2}\left(\Gamma_{\Sigma}\right) \\
q & \mapsto q \circ \Phi,
\end{aligned}
$$

and their continuous extension

$$
\begin{gathered}
A_{-1 / 2}: \quad\left[H^{1 / 2}\left(\Gamma_{\Omega}\right)\right]^{*} \rightarrow\left[H^{1 / 2}\left(\Gamma_{\Sigma}\right)\right]^{*} \\
\gamma \mapsto \gamma \circ \Phi
\end{gathered}
$$

are bounded and have bounded inverses.

\section{Proof.}

(a) The proof of the first claim is given in ([25], Chap. 2, Lem. 3.2), see also [19]. We denote the operator norms by $\left\|A_{1}\right\|$ and $\left\|A_{1}^{-1}\right\|$. The corresponding transformation formula is stated in Chapter 3.3 , Theorem 2 from [17].

(b) We show the boundedness of $A_{1 / 2}$. Note that we can write the operator in terms of $A_{1}$ and trace operators,

$$
H^{1 / 2}\left(\Gamma_{\Omega}\right) \stackrel{\text { inverse }}{\longrightarrow} H^{1}(\Omega) \stackrel{A_{1}}{\longrightarrow} H^{1}(\Sigma) \stackrel{\text { trace }}{\longrightarrow} H^{1 / 2}\left(\Gamma_{\Sigma}\right)
$$

Then, the boundedness of $A_{1}$, the trace operator ([31], Thm. 2.21), and the inverse trace operator ([31], Thm. 2.22), imply

$$
\left\|A_{1 / 2}\right\| \leq C_{\operatorname{tr}(\Omega)}\left\|A_{1}\right\| C_{\text {invTr }(\Sigma)} .
$$

(c) It follows from density arguments that the standard transformation formula on $\Gamma_{\Sigma}$ remains true for $f \in$ $H^{1 / 2}\left(\Gamma_{\Omega}\right)$, i.e.,

$$
\int_{\Gamma_{\Sigma}} f(\Phi(x)) \mathrm{d} x=\int_{\Gamma_{\Omega}=\Phi\left(\Gamma_{\Sigma}\right)} f(y)\left|\operatorname{det} \mathrm{D} \Phi^{-1}(y)\right| \mathrm{d} y .
$$


As an extension of $A_{1 / 2}$, the operator $A_{-1 / 2}$ is defined for $\gamma \in\left[H^{1 / 2}\left(\Gamma_{\Omega}\right)\right]^{*}$ as the limit

$$
A_{-1 / 2} \gamma=\lim _{j \rightarrow \infty} A_{1 / 2} \gamma_{j}
$$

for a sequence $\left\{\gamma_{j}\right\} \subset H^{1 / 2}\left(\Gamma_{\Omega}\right)$ with $\gamma_{j} \rightarrow \gamma$ in $\left[H^{1 / 2}\left(\Gamma_{\Omega}\right)\right]^{*}$. By the transformation formula (2.5) and part (b) of this theorem, we obtain

$$
\begin{aligned}
\left\|A_{-1 / 2} \gamma_{j}\right\|_{-1 / 2, \Gamma_{\Sigma}} & =\left\|\gamma_{j} \circ \Phi\right\|_{-1 / 2, \Gamma_{\Sigma}} \\
& =\sup _{p \in H^{1 / 2}\left(\Gamma_{\Sigma}\right)} \frac{\int_{\Gamma_{\Sigma}}\left(\gamma_{j} \circ \Phi\right) \cdot p \mathrm{~d} x}{\|p\|_{1 / 2, \Gamma_{\Sigma}}} \\
& \leq\left\|A_{1 / 2}^{-1}\right\| \sup _{p \in H^{1 / 2}\left(\Gamma_{\Sigma}\right)} \frac{\int_{\Gamma_{\Sigma}}\left(\gamma_{j} \cdot\left(p \circ \Phi^{-1}\right)\right) \circ \Phi \mathrm{d} x}{\left\|p \circ \Phi^{-1}\right\|_{1 / 2, \Gamma_{\Omega}}} \\
& =\left\|A_{1 / 2}^{-1}\right\| \sup _{q \in H^{1 / 2}\left(\Gamma_{\Omega}\right)} \frac{\int_{\Gamma_{\Sigma}}\left(\gamma_{j} \cdot q\right) \circ \Phi \mathrm{d} x}{\|q\|_{1 / 2, \Gamma_{\Omega}}} \\
& =\left\|A_{1 / 2}^{-1}\right\| \sup _{q \in H^{1 / 2}\left(\Gamma_{\Omega}\right)} \frac{\int_{\Gamma_{\Omega}} \gamma_{j} \cdot q\left|\operatorname{det} \mathrm{D} \Phi^{-1}\right| \mathrm{d} y}{\|q\|_{1 / 2, \Gamma_{\Omega}}} \\
& =\left\|A_{1 / 2}^{-1}\right\|\left\|\gamma_{j}\left|\operatorname{det} \mathrm{D} \Phi^{-1}\right|\right\|_{-1 / 2, \Gamma_{\Omega}} \\
& \leq\left\|A_{1 / 2}^{-1}\right\| c_{\Phi}^{-1}\left\|\gamma_{j}\right\|_{-1 / 2, \Gamma_{\Omega} .} .
\end{aligned}
$$

Thus, the operator $A_{-1 / 2}$ is bounded with constant $\left\|A_{1 / 2}^{-1}\right\| c_{\Phi}^{-1}$. The boundedness of the inverse operator $A_{-1 / 2}^{-1}$ follows by the same arguments.

The shown bi-Lipschitz equivalence from Theorem 2.5 is one of the main properties to proof the stability of the boundary constraint. The definition of the constraint operator and the proof of the inf-sup stability is subject of the remaining two subsections.

\subsection{Continuous inf-sup condition}

In order to include the boundary conditions as a weak constraint, we need a bilinear form which is defined on the moving boundary part $\Gamma_{D}(t)$. For this, we introduce for $t \in\left[t_{0}, T\right]$,

$$
b(\cdot, \cdot ; t): \mathcal{V} \times \mathcal{Q} \rightarrow \mathbb{R}
$$

With $v \in \mathcal{V}$, the bilinear form $b$ is densely defined, i.e., for $q \in\left[L^{2}(I)\right]^{d}$, by

$$
b(v, q ; t):=\int_{\Gamma_{D}(t)} v \cdot\left(q \circ \Phi^{-1}(t)\right) \mathrm{d} x .
$$

Note that $b$ is well-defined because of part (c) of Theorem 2.5. In the case of a fixed Dirichlet boundary, i.e., $\Phi(t, x)=\Phi(x)=x$ and $\Gamma_{D}(t)=\Gamma_{D}$, the bilinear form $b$ is independent of time and equals the bilinear form used in that setting $[2,29]$.

Remark 2.6. We could equivalently define $b$ with the transformation term $\left|\operatorname{det} \mathrm{D} \Phi^{-1}(t)\right|$. This corresponds to a scaling of the Lagrange multiplier and is used in [3] to model flexible multibody systems.

One important property of the bilinear form $b$ is the so-called inf-sup, LBB, or stability condition ([9], Chap. III.4). In the fixed boundary case, the inf-sup condition is easy to show with the help of the inverse trace theorem ([31], Thm. 2.22). The proof uses the fact that the dual of the ansatz space for the Lagrange multiplier equals the space of traces of $\mathcal{V}$. The situation in the time-dependent case is slightly changed since $\mathcal{Q}^{*}$ contains the traces of the transformed functions of $\mathcal{V}$. However, the proof of the stability condition of $b$ follows the same ideas ([31], Lem. 4.7). 
Lemma 2.7 (Inf-sup condition). Let $\Phi$ be the time-dependent bi-Lipschitz transformation from (2.2) satisfying (2.3) and Assumption 2.3. Then, the bilinear form b from (2.6) satisfies an inf-sup condition, i.e., there exists a positive constant $\beta$ such that for all $t \in\left[t_{0}, T\right]$,

$$
\inf _{q \in Q} \sup _{v \in \mathcal{V}} \frac{b(v, q ; t)}{\|v\|_{\mathcal{V}}\|q\|_{\mathcal{Q}}} \geq \beta>0 .
$$

Proof. Let $t \in\left[t_{0}, T\right]$ be arbitrary but fixed and let $\Sigma(t)$ be the inverse image of $\Omega$ under $\Phi(t)$. Consider an arbitrary element $q \in \mathcal{Q}$. Since the determinant of $\mathrm{D} \Phi(t)$ is bounded, it follows that $(q|\operatorname{det} \mathrm{D} \Phi(t)|) \in \mathcal{Q}$. According to the Riesz representation theorem ([31], Thm. 3.3), there exists an element $w(t) \in \mathcal{Q}^{*}$ such that for all $v \in \mathcal{Q}^{*}$,

$$
(w(t), v)_{\mathcal{Q}^{*}}=\langle q|\operatorname{det} \mathrm{D} \Phi(t)|, v\rangle_{\mathcal{Q}, \mathcal{Q}^{*}}
$$

Therein, $(\cdot, \cdot)_{\mathcal{Q}^{*}}$ denotes the inner product in $\mathcal{Q}^{*}$ and $\langle\cdot, \cdot\rangle_{\mathcal{Q}, \mathcal{Q}^{*}}$ the duality pairing given by the Gelfand triple from Remark 2.1. In addition, it holds that $\|w(t)\|_{\mathcal{Q}^{*}}=\|q|\operatorname{det} \mathrm{D} \Phi(t)|\|_{\mathcal{Q}}$. By the inverse trace theorem, there exists an extension of $w(t)$ on the domain $\Sigma(t)$. This extension, namely $v(t) \in\left[H^{1}(\Sigma(t))\right]^{d}$, satisfies

$$
\left.v(t)\right|_{I}=w(t)
$$

and, because of the uniform bound in $t$ by (2.4),

$$
\|v(t)\|_{1, \Sigma(t)} \leq C_{\mathrm{invTr}(\Sigma(t))}\|w(t)\|_{\mathcal{Q}^{*}} \leq C_{\mathrm{invTr}}\|w(t)\|_{\mathcal{Q}^{*}} .
$$

By the first part of Theorem 2.5, the transformation of $v(t)$ satisfies $\bar{v}(t):=v(t) \circ \Phi^{-1}(t) \in \mathcal{V}$. Thus, we can insert $\bar{v}(t)$ into the bilinear form $b$ and obtain by a sequence $\left\{q_{j}\right\} \subseteq\left[L^{2}(I)\right]^{d}$ with $q_{j} \rightarrow q$ in $\mathcal{Q}$ and the transformation formula (2.5),

$$
\begin{aligned}
\frac{b(\bar{v}(t), q ; t)}{\|\bar{v}(t)\|_{\mathcal{V}}} & =\lim _{j \rightarrow \infty} \frac{b\left(\bar{v}(t), q_{j} ; t\right)}{\|\bar{v}(t)\|_{\mathcal{V}}}=\lim _{j \rightarrow \infty} \frac{\left\langle q_{j}|\operatorname{det} \mathrm{D} \Phi(t)|, v(t)\right\rangle_{\mathcal{Q}, \mathcal{Q}^{*}}}{\|\bar{v}(t)\|_{\mathcal{V}}} \\
& =\frac{\langle q|\operatorname{det} \mathrm{D} \Phi(t)|, v(t)\rangle_{\mathcal{Q}_{\mathcal{Q}}}}{\|\bar{v}(t)\|_{\mathcal{V}}}=\frac{\langle q|\operatorname{det} \mathrm{D} \Phi(t)|, w(t)\rangle_{\mathcal{Q}, \mathcal{Q}^{*}}}{\|\bar{v}(t)\|_{\mathcal{V}}}=\frac{\|w(t)\|_{\mathcal{Q}^{*}}^{2}}{\|\bar{v}(t)\|_{\mathcal{V}}}
\end{aligned}
$$

With the first part of Theorem 2.5 and the inverse trace theorem, the norm of $\bar{v}(t)$ is bounded by

$$
\|\bar{v}(t)\|_{\mathcal{V}} \lesssim\|v(t)\|_{1, \Sigma(t)} \leq C_{\mathrm{invTr}}\|w(t)\|_{\mathcal{Q}^{*}}
$$

Furthermore, we can bound $\|w(t)\|_{\mathcal{Q}^{*}}$ from below with (2.3) by

$$
\|w(t)\|_{\mathcal{Q}^{*}}=\|q|\operatorname{det} \mathrm{D} \Phi(t)|\|_{\mathcal{Q}} \geq c_{\Phi}\|q\|_{\mathcal{Q}}
$$

All together, we yield the time-independent estimate

$$
\sup _{u \in \mathcal{V}} \frac{b(u, q ; t)}{\|u\|_{\mathcal{V}}} \geq \frac{b(\bar{v}(t), q ; t)}{\|\bar{v}(t)\|_{\mathcal{V}}} \gtrsim \frac{\|w(t)\|_{\mathcal{Q}^{*}}}{C_{\mathrm{invTr}}} \geq \frac{c_{\Phi}}{C_{\mathrm{invTr}}}\|q\|_{\mathcal{Q}}
$$

\subsection{Saddle point formulation}

With the bilinear form $b$ from (2.6) we are in the position to enforce the Dirichlet boundary conditions in a weak form. The needed Lagrange multiplier is defined on the time-independent domain $I$, as described in Section 2.1. The weak formulation in operator form reads: find $u \in L^{2}\left(t_{0}, T ; \mathcal{V}\right)$ with sufficiently smooth time derivatives and $\lambda \in L^{2}\left(t_{0}, T ; \mathcal{Q}\right)$ such that

$$
\begin{aligned}
& \mathcal{M} \ddot{u}(t)+\mathcal{D} \dot{u}(t)+\mathcal{K} u(t)+\mathcal{B}^{*}(t) \lambda(t)=\mathcal{F}(t) \quad \text { in } \quad \mathcal{V}^{*}, \\
& \mathcal{B}(t) u(t) \quad=\mathcal{G}(t) \quad \text { in } \quad \mathcal{Q}^{*}
\end{aligned}
$$


for a.e. $t \in\left[t_{0}, T\right]$ with initial conditions

$$
\begin{aligned}
& u\left(t_{0}\right)=g \in \mathcal{V}, \\
& \dot{u}\left(t_{0}\right)=h \in \mathcal{H} .
\end{aligned}
$$

Therein, $\mathcal{F}$ includes the applied forces and $\mathcal{G}$ contains the Dirichlet data $u_{D} \in H^{2}\left(t_{0}, T ; \mathcal{V}\right)$ and therefore the boundary conditions,

$$
\mathcal{G}(t):=b\left(u_{D}, \cdot ; t\right) \in \mathcal{Q}^{*}
$$

The time-dependent operator $\mathcal{B}(t): \mathcal{V} \rightarrow \mathcal{Q}^{*}$ and its dual $\mathcal{B}^{*}(t): \mathcal{Q} \rightarrow \mathcal{V}^{*}$ are defined via the bilinear form $b$ from $(2.6)$,

$$
\mathcal{B}(t) u:=b(u, \cdot ; t) \in \mathcal{Q}^{*}, \quad \mathcal{B}^{*}(t) \lambda:=b(\cdot, \lambda ; t) \in \mathcal{V}^{*} .
$$

Since (2.7) is an operator DAE, i.e., a DAE in an infinite dimensional setting, the initial values have to satisfy a consistency condition of the form $\mathcal{B}\left(t_{0}\right) g=\mathcal{G}\left(t_{0}\right)$. The precise definitions of the operators $\mathcal{M}$, $\mathcal{D}$, and $\mathcal{K}$ depend on the given problem. Note that the time derivatives in (2.7) should be understood in the generalized sense [34], Chapter 23.5.

An advantage of the formulation (2.7) is the time-independence of the spaces in which the equations are stated. It remains to show that the operator DAE (2.7) is well-posed in the sense that it is solvable if the corresponding PDE, constrained by Dirichlet boundary conditions on $\Gamma_{D}(t)$, is solvable.

Theorem 2.8 (Existence of the Lagrange multiplier). Consider operators $\mathcal{M}, \mathcal{D}$, and $\mathcal{K}$ with right-hand side $\mathcal{F} \in L^{2}\left(t_{0}, T ; \mathcal{V}^{*}\right)$. Further, let $u \in L^{2}\left(t_{0}, T ; \mathcal{V}\right)$ be a solution of

$$
\mathcal{M} \ddot{u}(t)+\mathcal{D} \dot{u}(t)+\mathcal{K} u(t)=\mathcal{F}(t),
$$

where the test functions from $\mathcal{V}$ vanish along $\Gamma_{D}(t)$ at time $t$, for given initial data $g, h$ and the constraint $u(t)=u_{D}(t)$ along $\Gamma_{D}(t)$ for a.e. $t \in\left[t_{0}, T\right]$. If the operators satisfy $\mathcal{M} \ddot{u}(t)+\mathcal{D} \dot{u}(t)+\mathcal{K} u(t) \in \mathcal{V}^{*}$ for a.e. $t \in\left[t_{0}, T\right]$, then there exists a unique $\lambda \in L^{2}\left(t_{0}, T ; \mathcal{Q}\right)$ such that $(u, \lambda)$ is a solution of $(2.7)$.

Proof. The claim follows directly from the inf-sup condition of Lemma 2.7 together with Theorem III.3.6 from [9] (see also [2], Thm. 4.11).

It remains the question of the existence of a solution $u$. For this, we give a particular existence result for the linear wave equation with moving Dirichlet conditions.

Example 2.9 (Linear wave equation). Consider the wave equation $\ddot{u}-\Delta u=f$, i.e., $\mathcal{M}=\mathrm{id}, \mathcal{D}=0$, and $\mathcal{K}$ corresponds to the Laplacian. Here, we assume that the transformation $\Phi$ has a time-independent preimage $\Sigma$, i.e., $\Phi(t, \Sigma)=\Omega$. Besides, we assume $\Phi(t)$ and its inverse to be continuously differentiable in $t$ and $\nabla(\operatorname{det} \mathrm{D} \Phi(t))$ to be uniformly bounded from above. Then, there exists a unique solution of (2.7), see Appendix A for a proof. For a numerical example, for which these assumptions are satisfied, we refer to [3].

\section{SEMi-DiscretizEd MODEL}

In this section, we analyse the saddle point formulation (2.7) after a semi-discretization in space. For this, we restrict ourselves to the two-dimensional case. For the discretization we use finite elements and need to introduce triangulations of $\Omega \subseteq \mathbb{R}^{2}$ as well as $I$. In the two-dimensional case, we may assume that $I \subset \mathbb{R}$ is an interval. The presented discretization scheme is stable in the sense that it satisfies a discrete inf-sup condition, which is crucial to ensure stable approximations of the Lagrange multiplier. 


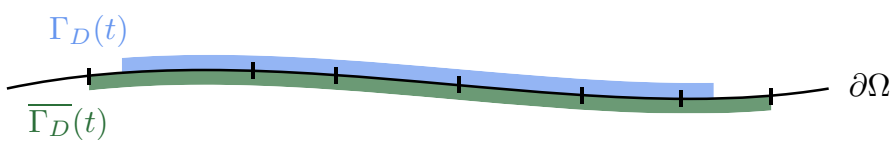

FiguRE 2. Illustration of the closure $\overline{\Gamma_{D}}(t)$ with respect to the triangulation $\mathcal{T}$.

\subsection{Finite element scheme}

Let $\mathcal{T}$ be a regular triangulation of $\Omega \subseteq \mathbb{R}^{2}$ in the sense of [14], i.e., we exclude hanging nodes. Furthermore, we assume $\mathcal{T}$ to be shape regular ([9], Chap. II.5). By $\mathcal{T}_{I}$ we denote a partition of the interval $I$. The set of edges of a triangulation or partition is denoted by $\mathcal{E}(\cdot)$.

In the sequel, we also need the partition of the moving boundary part which arises from the restriction of $\mathcal{T}$ on $\Gamma_{D}(t)$. This partition contains all edges of $\mathcal{T}$ which have a non-zero intersection with $\Gamma_{D}(t)$ in a one-dimensional measure,

$$
\mathcal{T}_{\Gamma}(t):=\left\{E \in \mathcal{E}(\mathcal{T}) \mid \operatorname{int}(E) \cap \Gamma_{D}(t) \neq \emptyset\right\} .
$$

With respect to this partition, we define the 'closure' of $\Gamma_{D}(t)$ by

$$
\overline{\Gamma_{D}}(t):=\bigcup_{E \in \mathcal{E}\left(\mathcal{T}_{\Gamma}(t)\right)} E .
$$

An illustrative picture of the closure is given in Figure 2. Clearly, it holds that $\Gamma_{D}(t) \subseteq \overline{\Gamma_{D}}(t)$ and they are equal if and only if the endpoints of $\Gamma_{D}(t)$ are nodes of the triangulation $\mathcal{T}$.

For the discretization in space, we introduce several finite element spaces. The space of piecewise polynomials of degree one which are globally continuous is denoted by

$$
\mathcal{S}_{h}:=\left[\mathcal{P}_{1}(\mathcal{T}) \cap C(\bar{\Omega})\right]^{d}=\operatorname{span}\left\{\varphi_{1}, \ldots, \varphi_{n_{1}}\right\} \subset \mathcal{V} .
$$

Therein, $\varphi_{1}, \ldots, \varphi_{n_{1}}$ denote the standard hat-functions ([9], Chap. II) in $d$ components and therefore a basis of $\mathcal{S}_{h}$. Thus, the dimension of $\mathcal{S}_{h}$ equals $d$ times the number of vertices in $\mathcal{T}$, namely $n_{1}$.

A second finite element space is given by edge-bubble functions as introduced in Chapter 1 from [32]. Here, we only consider edge-bubble functions on the boundary and in particular only edges which are part of $\overline{\Gamma_{D}}(t)$ at some point in time. Let $E_{1}, \ldots, E_{r} \in \mathcal{E}(\mathcal{T})$ denote these boundary edges, i.e.,

$$
\bigcup_{t \in\left[t_{0}, T\right]} \overline{\Gamma_{D}}(t)=\bigcup_{j=1}^{r} E_{j} .
$$

We define the space

$$
\mathcal{B}_{h}:=\operatorname{span}\left\{\psi_{1}, \ldots, \psi_{n_{2}}\right\} \subset \mathcal{V}
$$

where $n_{2}:=d \cdot r$ and $\psi_{1}, \ldots, \psi_{n_{2}}$ denote the standard edge-bubble functions in $d$ components for the $r$ boundary edges. Note that the dimension $n_{2}$ of the space $\mathcal{B}_{h}$ is independent of time. We summarize some properties of edge-bubble functions, which are important for later estimates. Recall that $\|\cdot\|_{0, T}$ and $\|\cdot\|_{0, E}$ denote the $L^{2}$-norm on a triangle $T$ and on an edge $E$, respectively.

Lemma 3.1 (Properties of edge-bubble functions). Let $\psi_{E}$ denote the edge-bubble function for a boundary edge $E$ of length $h=|E|$ and bordering triangle $T$, as shown in Figure 3. Furthermore, let $E$ be partitioned into two intervals $E_{1}, E_{2}$ with $\alpha:=\left|E_{1}\right| / h \geq 1 / 2$. Then,

(a) $\int_{E} \psi_{E} \mathrm{~d} x=2 h / 3$,

(b) $\left\|\nabla \psi_{E}\right\|_{0, T} \lesssim h^{-1 / 2}\|1\|_{0, E}$, 


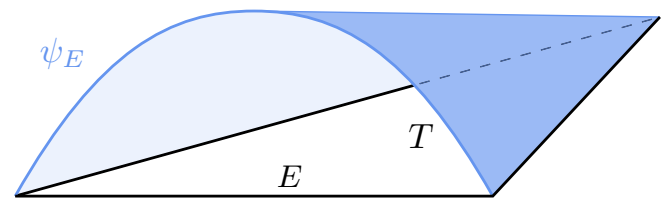

FiguRE 3. Illustration of an edge-bubble function $\psi_{E}$ corresponding to a boundary edge $E$.

The support of $\psi_{E}$ is given by the triangle $T$.

(c) $\int_{E_{1}} \psi_{E} \mathrm{~d} x \geq \alpha \int_{E} \psi_{E} \mathrm{~d} x$, and

(d) $\int_{E_{2}} \psi_{E} \mathrm{~d} x \leq(1-\alpha) \int_{E} \psi_{E} \mathrm{~d} x$.

The involved constant in (b) only depends on interior angles of the triangle $T$.

Proof. The first two claims are taken from Lemma 2.3.1 of [24]. The third claim follows by an easy calculation and the last claim follows directly from (c).

As finite dimensional approximation of the space $\mathcal{V}$, we use a combination of hat-functions and edge-bubble functions on the boundary,

$$
\mathcal{V}_{h}:=\mathcal{S}_{h} \oplus \mathcal{B}_{h}=\operatorname{span}\left\{\varphi_{1}, \ldots, \varphi_{n_{1}}, \psi_{1}, \ldots, \psi_{n_{2}}\right\} .
$$

The dimension of this space is given by $n:=n_{1}+n_{2}$. The ansatz space of the Lagrange multiplier $\mathcal{Q}$ is approximated by the space of piecewise constant functions on the interval $I$. For this, we introduce the functions $\chi_{i}$ which are constant along one edge of the partition $\mathcal{T}_{I}$ and vanish elsewhere. Since these ansatz functions are in $\left[L^{2}(I)\right]^{d}$, this provides a discontinuous but still conforming discretization,

$$
\mathcal{Q}_{h}:=\left[\mathcal{P}_{0}\left(\mathcal{T}_{I}\right)\right]^{d}=\operatorname{span}\left\{\chi_{1}, \ldots, \chi_{m}\right\} \subset \mathcal{Q} .
$$

The dimension of $\mathcal{Q}_{h}$, namely $m$, equals $d$ times the number of edges in $\mathcal{T}_{I}$. At this point, we assume $m<n$. As semi-discrete finite element approximations of $u$ and $\lambda$, we define

$$
u_{h}(t, x):=\sum_{j=1}^{n_{1}} q_{j}(t) \varphi_{j}(x)+\sum_{j=1}^{n_{2}} q_{n_{1}+j}(t) \psi_{j}(x), \quad \lambda_{h}(t, y):=\sum_{j=1}^{m} \mu_{j}(t) \chi_{j}(y) .
$$

The introduced discretization scheme also determines the positive definite $n$-by- $n$ mass matrix $M$, the damping matrix $D$, and the stiffness matrix $K$ as discrete representations of the operators $\mathcal{M}, \mathcal{D}$, and $\mathcal{K}$, respectively ([20], Chap. 12). For nonlinear operators, $D$ and $K$ may be replaced by some nonlinear functions. With $\varphi_{n_{1}+k}:=\psi_{k}$ for $k=1, \ldots, n_{2}$, the time-dependent $m$-by- $n$ coupling matrix $B(t)$ is given by

$$
B(t)_{j i}:=b\left(\varphi_{i}, \chi_{j} ; t\right)=\int_{\Gamma_{D}(t)} \varphi_{i} \cdot\left(\chi_{j} \circ \Phi^{-1}(t)\right) \mathrm{d} x .
$$

The described semi-discretization in space results in a DAE for the coefficient vectors $q=\left[q_{j}\right]$ and $\mu=\left[\mu_{j}\right]$,

$$
\begin{aligned}
M \ddot{q}(t)+D \dot{q}(t)+K q(t)+B^{T}(t) \mu(t) & =f(t), \\
B(t) q(t) & =g(t) .
\end{aligned}
$$

Because of the saddle point structure, the DAE (3.1) has (differentiation) index 3 if the matrix $B(t)$ is of full rank for all $t \in\left[t_{0}, T\right]$. For a precise definition of the index of a DAE (see [21], Chap. 3.3). Roughly speaking, the index gives the needed smoothness of the inhomogeneity to guarantee a continuously differentiable solution.

In the following subsection, we present assumptions under which the discretization scheme $\mathcal{V}_{h}-\mathcal{Q}_{h}$ fulfills a discrete inf-sup condition. The importance of this condition is commented in Chapter III.4 from [9]. Such a condition also implies the full rank property of $B(t)$ for all $t \in[0, T]$. 


\subsection{Discrete inf-sup condition}

In order to guarantee stability in the sense of a discrete inf-sup condition, the triangulations $\mathcal{T}_{\Gamma}(t)$ and $\mathcal{T}_{I}$ have to be compatible in the sense that the partition of $I$ is not too fine. A more precise formulation is given in the following assumption. Therein, $h_{I} \in L^{2}(I)$ and $h_{\Gamma} \in L^{2}\left(\overline{\Gamma_{D}}(t)\right)$ denote the piecewise constant functions which involve the local mesh-sizes, i.e.,

$$
\left.h_{I}\right|_{E}(x):=|E| \text { for } \quad E \subseteq I,\left.\quad h_{\Gamma}\right|_{F}(x):=|F| \text { for } \quad F \subseteq \overline{\Gamma_{D}}(t) .
$$

Assumption 3.2 (Compatibility of $\mathcal{T}_{\Gamma}(t)$ and $\mathcal{T}_{I}$ ). We assume that there exists a constant $0<\varepsilon<1 / 4$, independent of $t$, such that

$$
c_{\Phi}\left(h_{I} \circ \Phi^{-1}(t)\right) \geq(3 / 2+\varepsilon) h_{\Gamma}
$$

is satisfied a.e. on $\Gamma_{D}(t)$ with constant $c_{\Phi}$ from (2.3). The condition $\varepsilon<1 / 4$ is just included in order to unify the computations below.

The assumption states that the mesh-size of $\mathcal{T}_{I}$, transformed to $\Gamma_{D}(t)$, should be larger than the mesh-size of $\mathcal{T}$ along the moving boundary. In addition $\mathcal{T}_{\Gamma}(t)$ has to be quasi-uniform in the following sense.

Assumption 3.3 (Quasi-uniformity of $\left.\mathcal{T}_{\Gamma}(t)\right)$. Let $\kappa$ denote the largest ratio of two adjacent edges in the partition $\mathcal{T}_{\Gamma}(t)$. Then, we assume that $\kappa \leq 2$.

Remark 3.4. If the triangulation on the boundary $\mathcal{T}_{\Gamma}(t)$ is uniform, i.e., $\kappa=1$, then Assumption 3.2 can be weakened to $c_{\Phi}\left(h_{I} \circ \Phi^{-1}(t)\right) \geq(1+\varepsilon) h_{\Gamma}$.

In preparation for the main result of this section, we need to construct for a given function $\lambda_{h} \in \mathcal{Q}_{h}$ a piecewise constant function $\gamma_{h} \in\left[\mathcal{P}_{0}\left(\mathcal{T}_{\Gamma}(t)\right)\right]^{d}$ which is a good approximation of $\lambda_{h} \circ \Phi^{-1}(t)$. The construction of $\gamma_{h}$ is only necessary for the analysis of the finite element scheme and does not have to be computed in the actual simulation. To clarify the notation, in the sequel we neglect the time dependence of $\Phi^{-1}$.

\subsubsection{Construction of $\gamma_{h}$}

Assume that $t \in\left[t_{0}, T\right]$ is arbitrary but fixed. Consider $\lambda_{h} \in \mathcal{P}_{0}\left(\mathcal{I}_{I}\right)$ and its transformed analogon $\lambda_{h} \circ \Phi^{-1}$, which is also piecewise constant and hence in $L^{2}\left(\Gamma_{D}(t)\right)$. Without relabeling, we extend this function by zero such that $\lambda_{h} \circ \Phi^{-1} \in L^{2}\left(\overline{\Gamma_{D}}(t)\right)$. Note that $\lambda_{h} \circ \Phi^{-1}$ is piecewise constant but not necessarily with respect to $\mathcal{T}_{\Gamma}(t)$, i.e., $\lambda_{h} \circ \Phi^{-1} \notin \mathcal{P}_{0}\left(\mathcal{T}_{\Gamma}(t)\right)$.

Proposition 3.5. Under Assumption 3.2 or the weaker condition of Remark 3.4, the piecewise constant function $\lambda_{h} \circ \Phi^{-1}$ can only take two different values on an edge $E \in \mathcal{E}\left(\mathcal{T}_{\Gamma}(t)\right)$.

Proof. Suppose that $\lambda_{h} \circ \Phi^{-1}$ has more than two values on $E$. Then, there exists an edge $F \in \mathcal{E}\left(\mathcal{T}_{I}\right)$ with $\Phi(F) \subset E$ and $|\Phi(F)|<|E|$. Equation (2.3) then implies that for $x \in \Phi(F)$,

$$
c_{\Phi}\left(h_{I} \circ \Phi^{-1}\right)(x)=c_{\Phi}|F| \leq|\Phi(F)|<|E|=h_{\Gamma}(x)
$$

which is a contradiction to Assumption 3.2 as well as Remark 3.4.

We define the approximation of $\lambda_{h} \circ \Phi^{-1}$ in $\mathcal{P}_{0}\left(\mathcal{T}_{\Gamma}(t)\right)$ edge-wise. For this, consider an edge $E \in \mathcal{E}\left(\mathcal{T}_{\Gamma}(t)\right)$ with a partition $E=E_{1} \cup E_{2}$ such that

$$
\left.\lambda_{h} \circ \Phi^{-1}\right|_{E}(x)=\left\{\begin{array}{lll}
\alpha & \text { for } & x \in E_{1}, \\
\beta & \text { for } & x \in E_{2} .
\end{array}\right.
$$


Such a partition always exists because of Proposition 3.5. If $\lambda_{h} \circ \Phi^{-1}$ is constant on $E$, then $E_{2}$ vanishes. With the help of this decomposition, we define $\gamma_{h} \in \mathcal{P}_{0}\left(\mathcal{T}_{\Gamma}(t)\right)$ by

$$
\left.\gamma_{h}\right|_{E}:= \begin{cases}\alpha & \text { if }\left|E_{1}\right| \geq\left|E_{2}\right| \\ \beta & \text { otherwise. }\end{cases}
$$

Before we show that $\gamma_{h}$ is a reasonable approximation of $\lambda_{h} \circ \Phi^{-1}$, we define weighted norms of $L^{2}\left(\Gamma_{D}(t)\right)$ and $L^{2}\left(\overline{\Gamma_{D}}(t)\right)$,

$$
\|\cdot\|_{h}^{2}:=\sum_{E \in \mathcal{E}\left(\mathcal{T}_{\Gamma}(t)\right)} h_{E}\|\cdot\|_{L^{2}\left(E \cap \Gamma_{D}(t)\right)}^{2}, \quad\|\cdot\|_{h, \bar{\Gamma}}^{2}:=\sum_{E \in \mathcal{E}\left(\mathcal{T}_{\Gamma}(t)\right)} h_{E}\|\cdot\|_{L^{2}(E)}^{2} \cdot
$$

The approximation property of the constructed function $\gamma_{h}$ in (3.4) is given in the following lemma.

Lemma 3.6 (Approximation property of $\gamma_{h}$ ). Let $\psi_{E}$ denote the edge-bubble function for an edge $E$ and consider $\lambda_{h} \in \mathcal{P}_{0}\left(\mathcal{T}_{I}\right)$ as well as Assumptions 3.2 and 3.3. Then, the corresponding function $\gamma_{h} \in \mathcal{P}_{0}\left(\mathcal{T}_{\Gamma}(t)\right)$ defined in (3.4) satisfies

$$
\sum_{E \in \mathcal{E}\left(\mathcal{T}_{\Gamma}(t)\right)} h_{E} \int_{E \cap \Gamma_{D}(t)}\left(\lambda_{h} \circ \Phi^{-1}\right) \cdot \gamma_{h} \psi_{E} d x \geq \frac{\varepsilon}{4} \sum_{E \in \mathcal{E}\left(\mathcal{T}_{\Gamma}(t)\right)} h_{E} \int_{E} \gamma_{h}^{2} \psi_{E} d x
$$

and

$$
\left\|\lambda_{h} \circ \Phi^{-1}\right\|_{h} \leq \sqrt{3}\left\|\gamma_{h}\right\|_{h, \bar{\Gamma}}
$$

Proof. Recall that $0<\varepsilon<1 / 4$ and that $h_{E}=|E|$ denotes the length of an edge $E$. With the partition of $E$ as in (3.3), we distinguish two types of edges:

type 1: $\lambda_{h} \circ \Phi^{-1}$ is constant along $E$, i.e., $E=E_{1}$,

type 2: $\quad \lambda_{h} \circ \Phi^{-1}$ takes two different values, i.e., $\left|E_{2}\right| \neq 0$ and $\left|E_{1}\right| \geq h_{E} / 2$.

Consider an arbitrary edge $E \in \mathcal{E}\left(\mathcal{T}_{\Gamma}(t)\right)$ with $E \subseteq \Gamma_{D}(t)$. If $E$ is of first type, then

$$
h_{E} \int_{E}\left(\lambda_{h} \circ \Phi^{-1}\right) \cdot \gamma_{h} \psi_{E} \mathrm{~d} x=h_{E} \int_{E} \gamma_{h}^{2} \psi_{E} \mathrm{~d} x .
$$

If $E$ is of second type, we obtain with parts (c) and (d) of Lemma 3.1 and Young's inequality $2 a b \leq \sigma a^{2}+b^{2} / \sigma$ for $\sigma>0$ ([16], Appendix B) that

$$
\begin{aligned}
h_{E} \int_{E}\left(\lambda_{h} \circ \Phi^{-1}\right) \cdot \gamma_{h} \psi_{E} \mathrm{~d} x & =h_{E} \int_{E_{1}} \alpha^{2} \psi_{E} \mathrm{~d} x+h_{E} \int_{E_{2}} \alpha \beta \psi_{E} \mathrm{~d} x \\
& \geq \frac{h_{E}}{2} \int_{E} \alpha^{2} \psi_{E} \mathrm{~d} x-\frac{h_{E}}{2 \sigma} \int_{E_{2}} \alpha^{2} \psi_{E} \mathrm{~d} x-\frac{h_{E} \sigma}{2} \int_{E_{2}} \beta^{2} \psi_{E} \mathrm{~d} x \\
& \geq \frac{h_{E}}{4}\left(2-\frac{1}{\sigma}\right) \int_{E} \gamma_{h}^{2} \psi_{E} \mathrm{~d} x-\frac{h_{F} \sigma \kappa^{2}}{4} \int_{F} \gamma_{h}^{2} \psi_{F} \mathrm{~d} x .
\end{aligned}
$$

Thereby, $F$ denotes the edge adjacent of $E_{2}$ as shown in Figure 4 . With the choice $\sigma=1 /(\kappa-\varepsilon)$, we obtain an estimate of the form

$$
h_{E} \int_{E}\left(\lambda_{h} \circ \Phi^{-1}\right) \cdot \gamma_{h} \psi_{E} \mathrm{~d} x \geq c_{1}(\varepsilon) h_{E} \int_{E} \gamma_{h}^{2} \psi_{E} \mathrm{~d} x-c_{2}(\varepsilon) h_{F} \int_{F} \gamma_{h}^{2} \psi_{F} \mathrm{~d} x .
$$




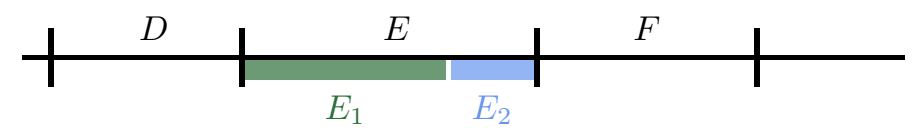

Figure 4 . An edge $E$, partitioned into $E_{1}$ and $E_{2}$, with neighboring edges $D$ and $F$ as in the proof of Lemma 3.6.

Because of Assumption 3.3, the constants $c_{1}$ and $c_{2}$ satisfy

$$
c_{1}(\varepsilon)=\frac{1}{4}(2-\kappa+\varepsilon) \geq \frac{\varepsilon}{4}, \quad c_{2}(\varepsilon)=\frac{\kappa^{2}}{4(\kappa-\varepsilon)} .
$$

For an edge $E$ with $E \nsubseteq \Gamma_{D}(t)$, i.e., an edge with only one neighbor in $\overline{\Gamma_{D}(t)}$ (recall Fig. 2), it holds that

$$
\int_{E \cap \Gamma_{D}(t)}\left(\lambda_{h} \circ \Phi^{-1}\right) \cdot \gamma_{h} \psi_{E} \mathrm{~d} x \geq \frac{1}{2} \int_{E} \gamma_{h}^{2} \psi_{E} \mathrm{~d} x .
$$

We are now in the position to sum up all contributions which gives

$$
\sum_{E \in \mathcal{E}\left(\mathcal{T}_{\Gamma}(t)\right)} h_{E} \int_{E \cap \Gamma_{D}(t)}\left(\lambda_{h} \circ \Phi^{-1}\right) \cdot \gamma_{h} \psi_{E} \mathrm{~d} x \geq \sum_{E \in \mathcal{E}\left(\mathcal{T}_{\Gamma}(t)\right)} c_{E} h_{E} \int_{E} \gamma_{h}^{2} \psi_{E} \mathrm{~d} x .
$$

It remains to show $c_{E} \geq \varepsilon / 4$ for all edges $E \in \mathcal{E}\left(\mathcal{T}_{\Gamma}(t)\right)$. Because of (3.2), negative contributions can only arise for edges of first type. Thus, it holds that $c_{E} \geq c_{1}(\varepsilon) \geq \varepsilon / 4$ for edges $E$ of second type or $E \nsubseteq \Gamma_{D}(t)$. If $E$ is of first type, we distinguish two cases: First, there is only one negative contribution coming from a neighboring edge in form of (3.7). Then, with (3.6) we obtain the estimate

$$
c_{E}=1-c_{2}(\varepsilon)=1-\frac{1}{4} \frac{\kappa^{2}}{\kappa-\varepsilon} \geq 1-\frac{1}{2-\varepsilon}>\frac{3}{7} .
$$

In the second case, we have two negative terms for the edge $E$, i.e., there are negative contributions from both neighboring edges. We show that (3.2) then locally implies a stricter bound on $\kappa$. Let $D$ and $F$ denote the neighboring edges of $E$ as illustrated in Figure 4 (here with $E=E_{1}$ ) and $D_{2}, F_{2}$ the adjacent parts, respectively. The restriction of the mesh-size (3.2) implies $\left|D_{2}\right| \leq|D| / 2=h_{D} / 2$ and $\left|F_{2}\right| \leq|F| / 2=h_{F} / 2$. Locally, the largest ratio of two adjacent edges is given by the maximum of the ratios $h_{D} / h_{E}$ and $h_{F} / h_{E}$. We assume w.l.o.g. that $h_{D} \geq h_{F}$ and thus, obtain the local edge ratio $\kappa_{E}:=h_{D} / h_{E}$. Then, Assumption 3.2 implies

$$
(3 / 2+\varepsilon) \kappa_{E} h_{E}=(3 / 2+\varepsilon) h_{D}<\left.c_{\Phi}\left(h_{I} \circ \Phi\right)\right|_{D_{2}} \leq\left|D_{2}\right|+|E|+\left|F_{2}\right| \leq\left(1+\kappa_{E}\right) h_{E} .
$$

Thus, $\kappa_{E}<2 /(1+2 \varepsilon)$ which leads to

$$
c_{E}=1-2 c_{2}(\varepsilon)=1-\frac{1}{2} \frac{\kappa_{E}^{2}}{\kappa_{E}-\varepsilon} \geq 1-\frac{2}{(1+2 \varepsilon)\left(2-\varepsilon-2 \varepsilon^{2}\right)} \geq \frac{\varepsilon}{4} .
$$

In total, this yields the stated estimate (3.5a).

For the second claim (3.5b), consider an arbitrary edge $E \in \mathcal{E}\left(\mathcal{T}_{\Gamma}(t)\right)$. If $\lambda_{h} \circ \Phi^{-1}$ is constant along $E$, then $\left\|\lambda_{h} \circ \Phi^{-1}\right\|_{L^{2}(E)}=\left\|\gamma_{h}\right\|_{L^{2}(E)}$. Otherwise, we distinguish between the cases $E \subseteq \Gamma_{D}(t)$ and $E \nsubseteq \Gamma_{D}(t)$. In the first case, we have (w.l.o.g. $\left|E_{2}\right| \leq\left|E_{1}\right|$ )

$$
\left\|\lambda_{h} \circ \Phi^{-1}\right\|_{L^{2}(E)}^{2}=\int_{E_{1}} \alpha^{2} \mathrm{~d} x+\int_{E_{2}} \beta^{2} \mathrm{~d} x \leq \int_{E} \alpha^{2} \mathrm{~d} x+\frac{\kappa}{2} \int_{F} \beta^{2} \mathrm{~d} x \leq\left\|\gamma_{h}\right\|_{L^{2}(E \cup F)}^{2} .
$$


Therein, $F$ denotes the neighboring edge of $E$ on which $\gamma_{h}$ takes the value $\beta$. For a boundary edge, $\gamma_{h}$ either equals the value of $\lambda_{h} \circ \Phi^{-1}$,

$$
\left\|\lambda_{h} \circ \Phi^{-1}\right\|_{L^{2}\left(E \cap \Gamma_{D}(t)\right)}^{2}=\left\|\gamma_{h}\right\|_{L^{2}\left(E \cap \Gamma_{D}(t)\right)}^{2} \leq\left\|\gamma_{h}\right\|_{L^{2}(E)}^{2}
$$

or vanishes along $E$. Then, again with neighboring edge $F$,

$$
\left\|\lambda_{h} \circ \Phi^{-1}\right\|_{L^{2}\left(E \cap \Gamma_{D}(t)\right)}^{2} \leq\left\|\gamma_{h}\right\|_{L^{2}(E \cup F)}^{2} .
$$

The summation over all edges finally proves the claim.

\subsubsection{Proof of the discrete inf-sup condition}

With the approximation $\gamma_{h}$ from (3.4) in hand, we are able to proof the stability condition of the discretization scheme $\mathcal{V}_{h}-\mathcal{Q}_{h}$ introduced in Section 3.1.

Theorem 3.7 (Discrete inf-sup condition). Under Assumptions 2.3, 3.2 and 3.3, the bilinear form $b$ from (2.6) satisfies a discrete inf-sup condition w.r.t. the discrete spaces $\mathcal{V}_{h}$ and $\mathcal{Q}_{h}$, i.e., there exists a positive constant $\beta_{\text {disc }}(\varepsilon)$, independent of the mesh-sizes and time, with

$$
\inf _{\lambda_{h} \in \mathcal{Q}_{h}} \sup _{v_{h} \in \mathcal{V}_{h}} \frac{b\left(v_{h}, \lambda_{h} ; t\right)}{\left\|v_{h}\right\| \mathcal{V}\left\|\lambda_{h}\right\|_{\mathcal{Q}}} \geq \beta_{\text {disc }}(\varepsilon)>0 .
$$

Proof. The proof basically works as for a fixed Dirichlet boundary ([24], Thm. 2.3.7). Nevertheless, the involved transformation requires several adjustments such that we give the details here.

Consider an arbitrary $\lambda_{h} \in \mathcal{Q}_{h}$ with $\left\|\lambda_{h}\right\|_{-1 / 2, I}=1$. As in [24], we show the existence of constants $c_{1}, c_{2}, c_{3}$, which may depend on $\varepsilon$ but not on the mesh-size or time, such that

$$
\text { (i) } \sup _{v_{h} \in \mathcal{V}_{h}} \frac{b\left(v_{h}, \lambda_{h} ; t\right)}{\left\|v_{h}\right\|_{\mathcal{V}}} \geq c_{1}\left\|\lambda_{h} \circ \Phi^{-1}(t)\right\|_{h}, \quad \text { (ii) } \sup _{v_{h} \in \mathcal{V}_{h}} \frac{b\left(v_{h}, \lambda_{h} ; t\right)}{\left\|v_{h}\right\|_{\mathcal{V}}} \geq c_{2}-c_{3}\left\|\lambda_{h} \circ \Phi^{-1}(t)\right\|_{h} .
$$

Since $\|\cdot\|_{h} \geq 0$, the claim then follows from

$$
\sup _{v_{h} \in \mathcal{V}_{h}} \frac{b\left(v_{h}, \lambda_{h} ; t\right)}{\left\|v_{h}\right\|_{\mathcal{V}}} \geq \max \left(c_{1}\left\|\lambda_{h} \circ \Phi^{-1}(t)\right\|_{h}, c_{2}-c_{3}\left\|\lambda_{h} \circ \Phi^{-1}(t)\right\|_{h}\right) \geq \frac{c_{1} c_{2}}{c_{1}+c_{2}} .
$$

In the proof we use generic constants which are independent of the mesh-size and time. Furthermore, we neglect the time-dependence of variables.

Proof of (i). As described at the beginning of this subsection, $\lambda_{h} \circ \Phi^{-1}$ can be extended by zero to a piecewise constant function on $\overline{\Gamma_{D}}(t)$. In addition, $\gamma_{h} \in\left[\mathcal{P}_{0}\left(\mathcal{T}_{\Gamma}(t)\right)\right]^{d}$ denotes the function defined componentwise as in (3.4). We define $u_{h} \in \mathcal{B}_{h} \subset \mathcal{V}_{h}$ by

$$
u_{h}:=\left.\sum_{E \in \mathcal{E}\left(\mathcal{T}_{\Gamma}(t)\right)} h_{E} \cdot \gamma_{h}\right|_{E} \cdot \psi_{E}
$$

with edge-bubble function $\psi_{E}$. Inserting $u_{h}$ into the bilinear form $b$, by Lemma 3.6 we obtain

$$
\begin{aligned}
b\left(u_{h}, \lambda_{h} ; t\right) & =\int_{\Gamma_{D}(t)} u_{h} \cdot\left(\lambda_{h} \circ \Phi^{-1}\right) \mathrm{d} x \\
& =\sum_{E \in \mathcal{E}\left(\mathcal{T}_{\Gamma}(t)\right)} h_{E} \int_{E \cap \Gamma_{D}(t)} \gamma_{h} \cdot \psi_{E} \cdot\left(\lambda_{h} \circ \Phi^{-1}(t)\right) \mathrm{d} x \\
& \geq \frac{\varepsilon}{4} \sum_{E \in \mathcal{E}\left(\mathcal{T}_{\Gamma}(t)\right)} h_{E} \int_{E} \gamma_{h}^{2} \cdot \psi_{E} \mathrm{~d} x=\frac{\varepsilon}{6} \sum_{E \in \mathcal{E}\left(\mathcal{T}_{\Gamma}(t)\right)} h_{E} \int_{E} \gamma_{h}^{2} \mathrm{~d} x=\frac{\varepsilon}{6}\left\|\gamma_{h}\right\|_{h, \bar{\Gamma}}^{2} .
\end{aligned}
$$


In the following, we use part (b) of Lemma 3.1. For a boundary edge $E$ we denote the adjacent triangle by $T_{E}$. By a Poincaré-Friedrichs inequality (e.g. [26] for convex domains), the $H^{1}$-norm of $u_{h}$ is bounded by

$$
\left\|u_{h}\right\|_{\mathcal{V}}^{2} \lesssim\left\|\nabla u_{h}\right\|_{0, \Omega}^{2}=\sum_{E \in \mathcal{E}\left(\mathcal{T}_{\Gamma}(t)\right)} h_{E}^{2}\left\|\nabla\left(\gamma_{h} \psi_{E}\right)\right\|_{0, T_{E}}^{2} \lesssim \sum_{E \in \mathcal{E}\left(\mathcal{T}_{\Gamma}(t)\right)} h_{E}\left\|\gamma_{h}\right\|_{0, E}^{2}=\left\|\gamma_{h}\right\|_{h, \bar{\Gamma}}^{2} .
$$

Together with the estimates (3.8) and (3.9), Lemma 3.6 yields

$$
\sup _{v_{h} \in \mathcal{V}_{h}} \frac{b\left(v_{h}, \lambda_{h} ; t\right)}{\left\|v_{h}\right\|_{\mathcal{V}}} \geq \frac{b\left(u_{h}, \lambda_{h} ; t\right)}{\left\|u_{h}\right\|_{\mathcal{V}}} \geq \frac{\varepsilon}{6} \frac{\left\|\gamma_{h}\right\|_{h, \bar{\Gamma}}^{2}}{\left\|u_{h}\right\|_{\mathcal{V}}} \gtrsim \varepsilon\left\|\gamma_{h}\right\|_{h, \bar{\Gamma}} \gtrsim \varepsilon\left\|\lambda_{h} \circ \Phi^{-1}\right\|_{h} .
$$

Proof of (ii): Note that Theorem 2.5 implies $\lambda_{h} \circ \Phi^{-1} \in\left[H^{1 / 2}\left(\Gamma_{D}(t)\right)^{*}\right]^{d}$ and

$$
1=\left\|\lambda_{h}\right\|_{-1 / 2, I} \leq\left\|A_{-1 / 2}\right\|\left\|\lambda_{h} \circ \Phi^{-1}\right\|_{-1 / 2, \Gamma_{D}(t)} .
$$

Thus, the norm of $\lambda_{h} \circ \Phi^{-1}$ is bounded from below. By the definition of the dual norm, there exists a $\hat{q} \in$ $\left[H^{1 / 2}\left(\Gamma_{D}(t)\right)\right]^{d}$ with $\|\hat{q}\|_{1 / 2, \Gamma_{D}(t)}=1$ such that

$$
\frac{1}{2\left\|A_{-1 / 2}\right\|} \leq \frac{1}{2}\left\|\lambda_{h} \circ \Phi^{-1}\right\|_{-1 / 2, \Gamma_{D}(t)} \leq \int_{\Gamma_{D}(t)} \hat{q} \cdot\left(\lambda_{h} \circ \Phi^{-1}\right) \mathrm{d} x .
$$

The rest of the proof then follows the lines of Theorem 2.3.7 from [24], using the weak solution of the Poisson equation with $\hat{q}$ as Dirichlet data. Finally, standard stability estimates yield with constants only depending on the minimal interior angle of the triangulation and $\left\|A_{-1 / 2}\right\|$,

$$
\sup _{v_{h} \in \mathcal{V}_{h}} \frac{b\left(v_{h}, \lambda_{h} ; t\right)}{\left\|v_{h}\right\|_{\mathcal{V}}} \geq \frac{b\left(\tilde{u}_{h}, \lambda_{h} ; t\right)}{\left\|\tilde{u}_{h}\right\|_{\mathcal{V}}} \gtrsim c_{2}-c_{3}\left\|\lambda_{h} \circ \Phi^{-1}\right\|_{h} .
$$

Remark 3.8 (Extension to the 3-dimensional case). The extension of the given stability result to domains $\Omega \subset \mathbb{R}^{3}$ seems possible since the underlying work [24] is valid in three dimensions. Nevertheless, the involved transformation causes several difficulties. The main difficulty is the absence of Proposition 3.5 in three dimensions. In this case, the number of different values one obtains on a boundary triangle may be arbitrary high, depending on the interior angles of the triangulation of $I$.

\section{Conclusion}

We have introduced a theoretical and numerical applicable framework to include Dirichlet boundary conditions on moving boundary parts. By initiating a time-dependent bi-Lipschitz transformation, we were able to formulate the dynamical system as a saddle point problem within time-independent ansatz spaces. One of the assumptions on the transformation is the boundedness of its derivative which causes a limitation of the length evolution of the boundary. Although the proofs work with the transformation of the entire domain, for practical computations it suffices to transform the Dirichlet boundary. Because of the saddle point structure, the key for the analysis of the continuous as well as the semi-discrete model is the verified inf-sup condition.

We have presented a spatial discretization scheme which is stable under some compatibility and quasiuniformity condition. The compatibility assumption is necessary to ensure that the number of constraints along the boundary is not larger than the number of degrees of freedom.

Possible fields of application include flexible multibody dynamics. In this context, Dirichlet boundary conditions may be used as dynamic coupling conditions. In order to stay within this framework, the coupling surfaces have to be known beforehand. This gives the main difference between this model and nonlinear contact problems. However, the framework is not restricted to model interconnections of flexible bodies. The presented model also allows to couple different kinds of physics. 


\section{Appendix A. Proof of Example 2.9}

The idea of the proof is to rewrite (2.7) such that a transformation by $\Phi$ leads to a fixed Dirichlet boundary problem. As a consequence, the transformed operator $\overline{\mathcal{K}}$ will be time-dependent. The stated result then follows from the general framework in Chapter V from [33]. In this part, we often neglect the time-dependence of the variables.

As a first step, we reformulate $(2.7)$ with the operator $\hat{\mathcal{B}}(t): \mathcal{V} \rightarrow \mathcal{Q}^{*}$, which is defined by

$$
\langle\hat{\mathcal{B}}(t) u, q\rangle_{\mathcal{Q}^{*}, \mathcal{Q}}:=\hat{b}(u, q ; t):=\int_{\Gamma_{D}(t)} u \cdot\left(q \circ \Phi^{-1}(t)\right)\left|\operatorname{det} \mathrm{D} \Phi^{-1}(t)\right| \mathrm{d} x .
$$

To obtain an equivalent system, we have to adjust the right-hand side $\mathcal{G}$ and $\lambda$, respectively. Therefore, we introduce $\langle\hat{\mathcal{G}}(t), q\rangle:=\hat{b}\left(u_{D}, q ; t\right)$ and define $\hat{\lambda} \in L^{2}\left(t_{0}, T ; \mathcal{Q}\right)$ by the one-to-one relation

$$
\hat{\lambda} \circ \Phi^{-1}\left|\operatorname{det} \mathrm{D} \Phi^{-1}\right|=\lambda \circ \Phi^{-1} \text {. }
$$

Thus, system (2.7) has a solution $(u, \lambda)$ if and only if there exists a solution of

$$
\begin{array}{rlrl}
\ddot{u}(t)+\mathcal{K} u(t)+\hat{\mathcal{B}}^{*}(t) \hat{\lambda}(t) & =\mathcal{F}(t) & \text { in } \quad \mathcal{V}^{*}, \\
\hat{\mathcal{B}}(t) u(t) & =\hat{\mathcal{G}}(t) & \text { in } \quad & \mathcal{Q}^{*}
\end{array}
$$

with the same initial values.

In the second step, we transform the equations by $\Phi$. For this, we use the variational formulation of (A.1). The transformation formula applied to (A.1b) then yields

$$
\int_{I}(u \circ \Phi) q \mathrm{~d} x=\langle\hat{\mathcal{G}}(t), q\rangle \quad \text { for all } \quad q \in \mathcal{Q} .
$$

Introducing $\varphi(t):=|\operatorname{det} \mathrm{D} \Phi(t)|$, which is bounded away from zero by (2.3), we obtain the equivalent equation

$$
\int_{I}\left(u \circ \Phi \varphi^{1 / 2}\right) q \varphi^{-1 / 2} \mathrm{~d} x=\left\langle\hat{\mathcal{G}}(t), q \varphi^{-1 / 2} \varphi^{1 / 2}\right\rangle=:\left\langle\overline{\mathcal{G}}(t), q \varphi^{-1 / 2}\right\rangle \quad \text { for all } q \in \mathcal{Q} .
$$

Defining $\bar{q}:=q \varphi^{-1 / 2} \in \mathcal{Q}$, we can equivalently test equation (A.2) for all $\bar{q} \in \mathcal{Q}$ instead of $q \in \mathcal{Q}$. Recall that $\Sigma$ denotes the preimage of $\Omega$ under $\Phi$, i.e., $\Phi(t, \Sigma)=\Omega$. Then, setting $a(t):=u(t) \circ \Phi(t) \varphi^{1 / 2}(t) \in \mathcal{V}_{\Sigma}:=\left[H^{1}(\Sigma)\right]^{d}$ (by Thm. 2.5), we obtain the equation

$$
\int_{I} a(t) \bar{q} \mathrm{~d} x=\langle\overline{\mathcal{G}}(t), \bar{q}\rangle \quad \text { for all } \quad \bar{q} \in \mathcal{Q}
$$

or, equivalently, in operator form with the time-independent trace operator $\overline{\mathcal{B}}: \mathcal{V}_{\Sigma} \rightarrow \mathcal{Q}^{*}$,

$$
\overline{\mathcal{B}} a(t)=\overline{\mathcal{G}}(t) \quad \text { in } \mathcal{Q}^{*} .
$$

The transformation formula applied to the variational formulation of (A.1a) yields

$$
\frac{\mathrm{d}^{2}}{\mathrm{~d} t^{2}}\left(u \circ \Phi \varphi^{1 / 2}, v \circ \Phi \varphi^{1 / 2}\right)_{\Sigma}+\langle\mathcal{K} u, v\rangle+\int_{I} v \circ \Phi \hat{\lambda} \mathrm{d} x=\langle\mathcal{F}(t), v\rangle \quad \text { for all } \quad v \in \mathcal{V} .
$$

Because of Theorem 2.5 and (2.3), the functions $w:=(v \circ \Phi) \varphi^{1 / 2}$ satisfy $w \in \mathcal{V}_{\Sigma}$. The bi-Lipschitz equivalence allows to test the equation with functions $w \in \mathcal{V}_{\Sigma}$ instead of $v \in \mathcal{V}$. Since we test with all functions in $\mathcal{V}_{\Sigma}$, we 
may assume that the test functions are independent of $t$. By a rescaling of $\hat{\lambda}$, which we denote by $\bar{\lambda}$, and the introduction of a new right-hand side $\overline{\mathcal{F}}$, we obtain the equivalent equation

$$
\frac{\mathrm{d}^{2}}{\mathrm{~d} t^{2}}(a(t), w)_{\Sigma}+\langle\overline{\mathcal{K}}(t) a(t), w\rangle+\langle\overline{\mathcal{B}} w, \bar{\lambda}\rangle=\langle\overline{\mathcal{F}}(t), w\rangle \quad \text { for all } \quad w \in \mathcal{V}_{\Sigma}
$$

or, as operator equation,

$$
\ddot{a}(t)+\overline{\mathcal{K}}(t) a(t)+\overline{\mathcal{B}}^{*} \bar{\lambda}(t)=\overline{\mathcal{F}}(t) \quad \text { in } \quad \mathcal{V}_{\Sigma}^{*} .
$$

Therein, the symmetric and time-dependent operator $\overline{\mathcal{K}}(t): \mathcal{V}_{\Sigma} \rightarrow \mathcal{V}_{\Sigma}^{*}$ is defined by

$$
\langle\overline{\mathcal{K}}(t) a, w\rangle_{\mathcal{V}_{\Sigma}^{*}, \mathcal{V}_{\Sigma}}:=\left\langle\mathcal{K}\left(\left(a \varphi^{-1 / 2}\right) \circ \Phi^{-1}\right),\left(w \varphi^{-1 / 2}\right) \circ \Phi^{-1}\right\rangle_{\mathcal{V}^{*}, \mathcal{V}}
$$

Since we have only used the transformation $\Phi$ and a rescaling by the bounded function $\varphi$, system (2.7) has a solution $(u, \lambda)$ if and only if there exists a solution $(a, \bar{\lambda})$ of (A.3)-(A.4).

In the last step we show that system (A.3)-(A.4) has a unique solution. Since the trace operator $\overline{\mathcal{B}}$ satisfies an inf-sup condition, by Theorem III.3.6 from [9], it is sufficient to show that the operator equation

$$
\ddot{a}(t)+\overline{\mathcal{K}} a(t)=\overline{\mathcal{F}}(t) \quad \text { in } \quad\left(\mathcal{V}_{\Sigma, I}\right)^{*}
$$

has a unique solution $a \in L^{2}\left(t_{0}, T ; \mathcal{V}_{\Sigma, I}\right)$. Thereby, $\mathcal{V}_{\Sigma, I}$ denotes the subspace of $\mathcal{V}_{\Sigma}$ with vanishing boundary values along $I \subset \partial \Sigma$. The in Example 2.9 assumed smoothness of the transformation $\Phi$ implies that the timedependent operator $\overline{\mathcal{K}}$ fits in the framework of Chapter $\mathrm{V}$ from [33]. Thus, the existence of a unique solution follows by [33], Theorem 29.1.

\section{REFERENCES}

[1] R.A. Adams and J.J.F. Fournier, Sobolev Spaces, 2nd edn. Elsevier, Amsterdam (2003).

[2] R. Altmann. Index reduction for operator differential-algebraic equations in elastodynamics. Z. Angew. Math. Mech. (ZAMM) 93 (2013) 648-664.

[3] R. Altmann. Modeling flexible multibody systems by moving Dirichlet boundary conditions. In Proc. of Multibody Dynamics 2013 - ECCOMAS Thematic Conference, Zagreb, Croatia, July 1-4 (2013).

[4] M. Arnold and B. Simeon, The simulation of pantograph and catenary: a PDAE approach. Preprint (1990), Technische Universität Darmstadt, Germany (1998).

[5] M. Arnold and B. Simeon, Pantograph and catenary dynamics: A benchmark problem and its numerical solution. Appl. Numer. Math. 34 (2000) 345-362.

[6] I. Babuška, The finite element method with Lagrangian multipliers. Numer. Math. 20 (1973) 179-192.

[7] I. Babuška and G.N. Gatica, On the mixed finite element method with Lagrange multipliers. Numer. Meth. Part. D. E. 19 (2003) 192-210,.

[8] F. Ben Belgacem, The mortar finite element method with Lagrange multipliers. Numer. Math. 84 (1999) $173-197$. Doi: $10.1007 / \mathrm{s} 002110050468$.

[9] D. Braess, Finite Elements - Theory, Fast Solvers, and Applications in Solid Mechanics, 3rd edn. Cambridge University Press, New York (2007).

[10] J.H. Bramble, The Lagrange multiplier method for Dirichlet's problem. Math. Comput. 37 (1981) 1-11.

[11] S. C. Brenner and L.R. Scott, The Mathematical Theory of Finite Element Methods, 3rd edn. Springer-Verlag, New York (2008).

[12] F. Brezzi and M. Fortin, Mixed and Hybrid Finite Element Methods. Springer-Verlag, New York (1991).

[13] F.J. Cavalieri, A. Cardona, V.D. Fachinotti and J. Risso, A finite element formulation for nonlinear 3D contact problems. Mecánica Comput. XXVI(16) (2007) 1357-1372.

[14] P.G. Ciarlet, The Finite Element Method for Elliptic Problems. North-Holland, Amsterdam (1978).

[15] E. Emmrich and D. Šiška, Evolution equations of second order with nonconvex potential and linear damping: existence via convergence of a full discretization. Technical report, University of Liverpool (2012).

[16] L.C. Evans, Partial Differential Equations, 2nd edn. American Mathematical Society (AMS). Providence (1998).

[17] L.C. Evans and R.F. Gariepy, Measure Theory and Fine Properties of Functions. Studies in Advanced Mathematics. CRC Press, Boca Raton, FL (1992). 
[18] M. Géradin and A. Cardona, Flexible Multibody Dynamics: A Finite Element Approach. John Wiley, Chichester (2001).

[19] J.A. Griepentrog, K. Gröger, H.-C. Kaiser and J. Rehberg, Interpolation for function spaces related to mixed boundary value problems. Math. Nachr. 241 (2002) 110-120.

[20] B. Gustafsson, High Order Difference Methods for Time Dependent PDE. Springer-Verlag, Berlin (2008).

[21] P. Kunkel and V. Mehrmann, Differential-Algebraic Equations: Analysis and Numerical Solution. European Mathematical Society (EMS), Zürich (2006).

[22] J.-L. Lions and E. Magenes, Problèmes aux Limites Non Homogènes et Applications. Vol. 1. Travaux et Recherches Mathématiques, No. 17. Dunod, Paris (1968).

[23] J.-L. Lions and W.A. Strauss, Some non-linear evolution equations. Bull. Soc. Math. France 93 (1965) $43-96$.

[24] M.K. Lipinski, A posteriori Fehlerschätzer für Sattelpunktsformulierungen nicht-homogener Randwertprobleme. Ph.D thesis, Ruhr Universität Bochum (2004).

[25] J. Nečas, Les Méthodes Directes en Théorie des Equations Elliptiques. Masson et Cie, Éditeurs, Paris (1967).

[26] L.E. Payne and H.F. Weinberger, An optimal Poincaré inequality for convex domains. Arch. Rational Mech. Anal. 5 (1960) $286-292$.

[27] G. Poetsch, J. Evans, R. Meisinger, W. Kortüm, W. Baldauf, A. Veitl and J. Wallaschek, Pantograph/catenary dynamics and control. Vehicle System Dynamics 28 (1997) 159-195.

[28] A.A. Shabana, Dynamics of Multibody Systems, 3rd edn. Cambridge University Press, Cambridge (2005).

[29] B. Simeon, On Lagrange multipliers in flexible multibody dynamics. Comput. Method. Appl. M 195 (2006) $6993-7005$.

[30] B. Simeon, Computational flexible multibody dynamics. A differential-algebraic approach. Differential-Algebraic Equations Forum. Springer-Verlag, Berlin (2013).

[31] O. Steinbach, Numerical Approximation Methods for Elliptic Boundary Value Problems: Finite and Boundary Elements. Springer-Verlag, New York (2008).

[32] R. Verfürth, A Review of A Posteriori Error Estimation and Adaptive Mesh-Refinement Techniques. Wiley-Teubner, Stuttgart (1996).

[33] J. Wloka, Partial Differential Equations. Cambridge University Press, Cambridge (1987).

[34] E. Zeidler, Nonlinear Functional Analysis and its Applications IIa: Linear Monotone Operators. Springer-Verlag, New York (1990). 